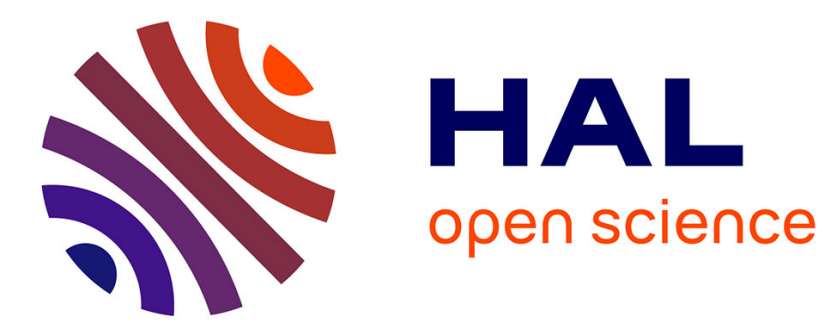

\title{
Elastocapillary flows in flexible tubes
}

\author{
Theresa B. Hoberg, Emilie Verneuil, A. E. Hosoi
}

\section{To cite this version:}

Theresa B. Hoberg, Emilie Verneuil, A. E. Hosoi. Elastocapillary flows in flexible tubes. Physics of Fluids, 2014, 26 (12), pp.122103. 10.1063/1.4902509 . hal-01441769

\section{HAL Id: hal-01441769 \\ https://hal.science/hal-01441769}

Submitted on 20 Jan 2017

HAL is a multi-disciplinary open access archive for the deposit and dissemination of scientific research documents, whether they are published or not. The documents may come from teaching and research institutions in France or abroad, or from public or private research centers.
L'archive ouverte pluridisciplinaire HAL, est destinée au dépôt et à la diffusion de documents scientifiques de niveau recherche, publiés ou non, émanant des établissements d'enseignement et de recherche français ou étrangers, des laboratoires publics ou privés. 


\section{Elastocapillary flows in flexible tubes}

Theresa B. Hoberg', Emilie Verneuil', and A. E. Hosoi'

Citation: Phys. Fluids 26, 122103 (2014); doi: 10.1063/1.4902509

View online: http://dx.doi.org/10.1063/1.4902509

View Table of Contents: http://aip.scitation.org/toc/phf/26/12

Published by the American Institute of Physics 


\title{
Elastocapillary flows in flexible tubes
}

\author{
Theresa B. Hoberg, ${ }^{1, a)}$ Emilie Verneuil, ${ }^{1,2, b)}$ and A. E. Hosoi ${ }^{1, c)}$ \\ ${ }^{1}$ Massachusetts Institute of Technology, 77 Massachusetts Ave., Cambridge, \\ Massachusetts 02139, USA \\ ${ }^{2}$ SIMM, UMR 7615, CNRS, UPMC, ESPCI ParisTech, 10 rue Vauquelin, \\ 75231 Paris, France
}

(Received 11 February 2014; accepted 4 November 2014; published online 12 December 2014)

\begin{abstract}
Interactions between capillary and elastic effects are relevant to a variety of applications from micro- and nano-scale manufacturing to biological systems. In this work, we investigate capillary flows in flexible, millimeter-scale cylindrical elastic tubes. We demonstrate that surface tension can cause sufficiently flexible tubes to collapse and coalesce spontaneously through non-axisymmetric buckling, and develop criteria for the initial deformation and complete collapse of a circular tube. Experimental results for capillary rise and evaporation of a liquid in a flexible tube are presented, and several regimes are seen for the equilibrium state of a flexible tube deforming under capillary pressure. Deformations of the tube walls are measured in different regimes and compared with a shell theory model. Analysis and experimental results show that despite the complex and non-axisymmetric deformed shapes of cylindrical structures, the elastocapillary length used in previous literature for flat plates and sheets can be used to predict the behavior of flexible tubes. (C) 2014 AIP Publishing LLC. [http://dx.doi.org/10.1063/1.4902509]
\end{abstract}

\section{INTRODUCTION}

Elastocapillary flows have generated considerable attention in the context of both engineered and natural systems. Interactions between surface tension and flexible structures are relevant to manufacturing at the micro- and nano-scale, where capillary effects can be detrimental if they deform a structure away from a goal geometry ${ }^{1}$ conversely these effects can be beneficial when used to build desirable structures. For example, the folding of a flat sheet into three dimensional shapes $^{2}$ and assembly of nanorods into hierarchical helical structures during evaporation of a liquid ${ }^{3}$ have been investigated, and are driven by elastocapillary interactions. Several biological systems, such as pulmonary airway closure ${ }^{4,5}$ and reopening of collapsed airways in the lungs, ${ }^{6}$ or the assembly of nanofibers on biological surfaces, ${ }^{7}$ also involve interactions between flexible structures and surface tension.

Figure 1 summarizes several regimes in which elasticity, capillarity, or a combination thereof drives dynamics. The summary is divided into flat structures (I) and structures with intrinsic curvature, or structures where three-dimensional effects are important (II). In this work, we focus on regime IId; however, a diverse array of literature-particularly in regimes Id and IIc as discussed below-can offer insight into our system. Results from the case of capillary rise in rigid systems (I,IIb) are briefly reviewed and compared with experimental data in the Appendix. Single-phase flow in rigid structures (I,IIa) is included in Figure 1 for completeness.

The 2010 topical review by Roman and Bico $^{8}$ presents an excellent overview of recent work on elastocapillary interactions and flows. Several configurations in regime Id have been studied, ${ }^{9-14}$ and it is well-known that for a flat plate or slender structure, a balance between the characteristic

\footnotetext{
a) thoberg@mit.edu

b)emilie.verneuil@espci.fr

c) peko@mit.edu
} 


\begin{tabular}{|c|c|c|c|c|c|}
\hline \multicolumn{3}{|c|}{ I. Flat Structures } & \multicolumn{3}{|c|}{ II. Curved Structures } \\
\hline & Rigid & Deformable & & Rigid & Deformable \\
\hline 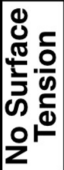 & $\begin{array}{l}\text { a. Planar flow } \\
\text { in a gap }\end{array}$ & $\begin{array}{l}\text { c. Flow past a } \\
\text { flexible membrane }\end{array}$ & 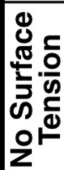 & $\begin{array}{l}\frac{\Theta}{\Theta} \mathbf{r}_{\downarrow} \\
\text { a. Pipe flow, } \\
\text { porous media }\end{array}$ & $\begin{array}{l}D \longrightarrow \\
\text { c. Starling resistor }\end{array}$ \\
\hline 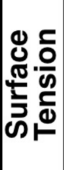 & $\begin{array}{l}\qquad \mathbf{2 b} G \\
\text { b. Planar } \\
\text { capillary flow }\end{array}$ & 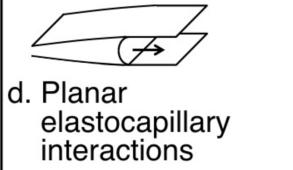 & 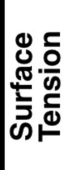 & $\begin{array}{l}\mathbf{r}_{\downarrow} \quad \rightarrow \\
\text { b. Capillary } \\
\text { flow in a tube, } \\
\text { porous media }\end{array}$ & $\begin{array}{l}\rightarrow \infty \infty \\
\text { d. Capillary flow } \\
\text { in a deformable } \\
\text { tube or porous } \\
\text { media }\end{array}$ \\
\hline
\end{tabular}

FIG. 1. Cases of interest for (I) flat sheets and slender structures ${ }^{2,8-15}$ and (II) curved structures. ${ }^{6,16-25}$

bending energy and capillary energy gives rise to an elastocapillary length scale, which characterizes the typical curvature that can be induced by capillarity. This elastocapillary length, $L_{e c}$, depends on the bending stiffness of the material, $B$, and the surface tension of the liquid, $\sigma$

$$
L_{e c}=\sqrt{\frac{B}{\sigma}}, \quad B=\frac{E h^{3}}{12\left(1-v^{2}\right)} .
$$

Here, $E$ is the elastic modulus, $h$ is the thickness of the sheet, and $v$ is the Poisson's ratio of the flexible sheet. In general, these studies of flexible flat sheets show that flexibility enhances capillary forces by deforming structures to reduce relevant characteristic length scales (e.g., by drawing parallel sheets together to reduce the interstitial gap).

In the absence of surface tension, fluid flow through flexible tubes (IIc) has been studied extensively, primarily in the context of biomechanics. The 2004 review by Grotberg and Jensen ${ }^{16}$ provides a comprehensive introduction to this topic. A typical experimental setup in this regime is known as a Starling resistor which consists of a flexible tube mounted between two rigid pipes, surrounded by an external pressure. Fluid flows through the tube, driven by an applied pressure drop. The shapes of cylindrical tubes deforming due to internal fluid flow have been studied both experimentally and theoretically, and several interesting instabilities and flow-structure interactions have been observed and analyzed. ${ }^{16-18}$

When modeling flows through these flexible tubes, an experimentally or theoretically determined relationship, known as the "tube law," is typically used to relate the deformed cross-sectional area to the pressure difference across the membrane, $\left(P-P_{a}\right)$, at each axial location. This problem has a rich history including work by Shapiro, ${ }^{17}$ who experimentally determined these tube shapes under different pressure differentials and Flaherty et al. ${ }^{26}$ who numerically simulated the shapes of tubes or rings after buckling, and found a similarity solution for the tube area after the point of opposite wall contact (see Sec. II).

Fewer studies have been performed on elastocapillary effects in a three-dimensional setting (regime IId). Capillary flows in deformable porous media ${ }^{22-24}$ - which are inherently threedimensional-and other complex geometries are relevant to geological flows, composites manufacturing, ${ }^{23}$ flows in biological tissues, and textiles engineering. ${ }^{24}$ Elastocapillary effects on flow in these cases may be significantly different from those in a planar structure as the deformation and coalescence process become more complex, and other length scales associated with the geometry of the structure may play a role.

The current study builds on previous studies performed in planar geometries and works toward addressing this three-dimensional complexity by examining elastocapillary flows in flexible cylindrical tubes. This system serves as an intermediate step toward porous media in geometrical complexity, while still allowing for detailed measurement and visualization. In flexible tubes, deformation is expected to take place through a non-axisymmetric, three-dimensional buckling process, hence the change in meniscus shape as the tube collapses is more complex than in the case of flat sheets. 
Several researchers have investigated the related problems of propagation of an air finger into a collapsed flexible tube, ${ }^{15,19,27}$ usually in the context of reopening an occluded lung vessel, and the collapse of liquid-lined flexible tubes due to surface tension, ${ }^{4,5,18,21}$ relevant to airway closure. These studies - which range in scale from carbon nano-tubes to airway reopening-tend to lie at extreme ends of the spectrum in terms of length scale, as characterized by the elastocapillary length. In contrast, the current experiments reside at an intermediate, millimetric, length scale.

There are a number of advantages associated with working at these millimetric scales. Compared to the nano-scale tubes, material and interface properties are relatively well known and experimental observation and visualization are more tractable. The flexible material used in the current experiments also allows for clear observation of post-buckling behavior. In contrast to previous experiments on airway reopening, gravity effects can be neglected in the current experiment since the tube radius is small compared to the capillary length of the system, and the meniscus takes on a well-defined shape. This condition may be expressed as

$$
r<L_{\text {cap }}=\sqrt{\frac{\sigma}{\rho g}} .
$$

Here, $r$ is the tube radius, $L_{c a p}$ is the capillary length, $\rho$ is the liquid density, and $g$ is the gravitational acceleration. If the tube is sufficiently flexible, surface tension is dominant in the open as well as the collapsed state. This allows for more straightforward comparison with theory and evaluation of simple models. Also, in the limit of tube diameters smaller than the capillary length, the hydrostatic pressure is negligible compared to the capillary term and the tubes can be suspended vertically, avoiding buoyancy effects across the tube.

\section{ANALYSIS: CAPILLARY FLOW IN FLEXIBLE TUBES}

The shape of a tube deforming under inward lateral pressure has been well studied both experimentally and theoretically. As shown in Figure 2, flexible tubes are expected to deform in

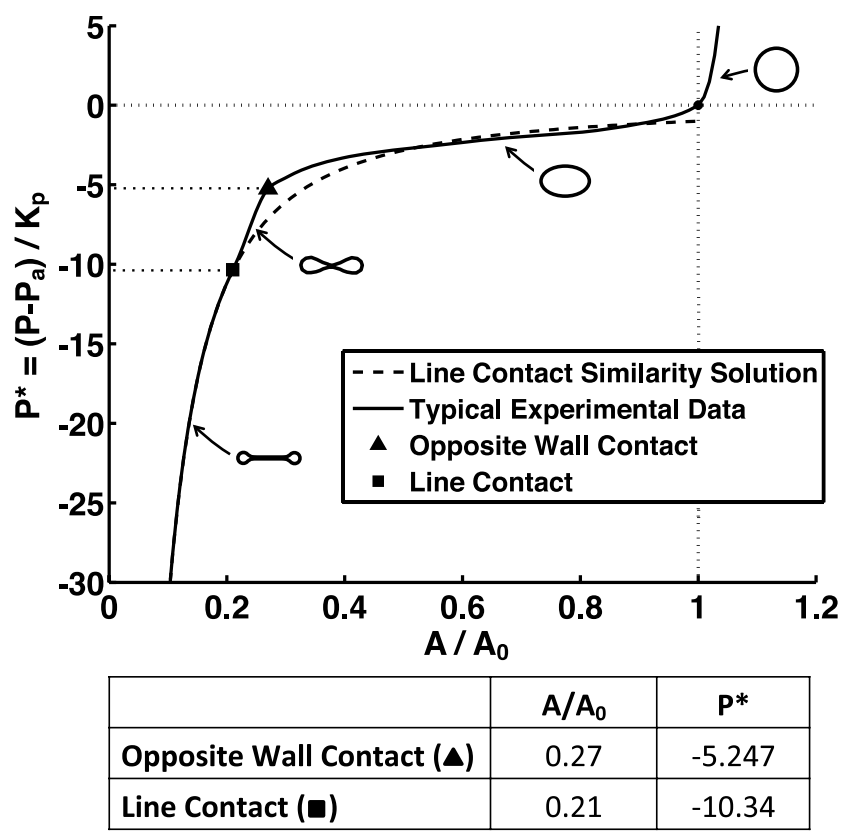

FIG. 2. Pressure versus area curve of a flexible tube. The solid black line schematically indicates typical experimental results, ${ }^{17,19}$ while the dashed black line indicates a similarity solution that is applicable in the line contact regime (Eq. (3)). Images of typical cross sections of a flexible tube are shown for the four primary regimes: circular, buckled, opposite wall contact, and line contact. Predicted transition points are given in the table. ${ }^{17,26}$ Here, $P$ represents the pressure inside the tube, $P_{a}$ is the surrounding or ambient pressure, and $A_{0}$ is the cross-sectional area of the undeformed tube. The normalizing parameter for pressure $K_{p}$ is given in Eq. (4). 
a complex, non-axisymmetric way, and to progress through three deformed shapes or stages. ${ }^{17,26}$ First, after a critical buckling pressure is reached, the tube will deform to an elliptical shape. As the inward pressure continues to increase, the cross-sectional area of the tube will continue to decrease until opposite walls contact at a point. Finally, this point of contact will reach zero curvature and start to extend into a line. This is usually modeled by a semi-empirical "tube law" relating area to pressure at each location.

In Figure 2, images show typical cross sections of a flexible tube for the four primary regimes: circular, buckled, opposite wall contact, and line contact. The solid black line schematically indicates typical experimental results. ${ }^{17,19}$ The pressure difference $\left(P-P_{a}\right)$ between the pressure inside the tube and the surrounding or ambient pressure can be non-dimensionalized by a factor $K_{p}$ (Eq. (4)) to give a non-dimensional pressure $P^{*}$, and the cross-sectional area of the deforming tube $A$ can be normalized by the cross-sectional area of the undeformed tube, $A_{0}$. Using a Poisson's ratio $v$ of 0.5 , the buckling transition is predicted to occur at $P^{*}=-2.25$, the transition to opposite wall contact at $P^{*}=-5.25$, and the transition to line contact at $P^{*}=-10.34$ for the case of a ring (see Sec. II A). In the line contact regime, a similarity solution developed by Flaherty et al. ${ }^{17,26}$ is applicable:

$$
\begin{gathered}
P^{*}=\left(\frac{P_{a}-P}{K_{p}}\right)=\left(\frac{A}{A_{0}}\right)^{-3 / 2}, \\
K_{p}=\frac{E h^{3}}{12\left(1-v^{2}\right) r^{3}} .
\end{gathered}
$$

The numerical predictions for transition to opposite wall contact and transition to line contact are shown in Figure 2. ${ }^{17,26}$

As seen from the slopes of the pressure versus area curve in Figure 2, once a tube has buckled, it becomes fairly compliant, and the area changes quickly with small changes in pressure until the point of opposite wall contact. Once opposite walls touch, however, the tube becomes more resistant to changes in area, due to the higher curvature and bending energy that build up at the two ends. ${ }^{16}$

\section{A. Buckling due to capillary pressure}

The initial buckling of a thin cylindrical shell to a non-circular shape, under inward lateral pressure, can be modeled using classical shell theory. ${ }^{28,29}$ The critical pressure for buckling of a "long" tube under field-pressure loading, or pressure normal to the tube wall at each point, is given by

$$
P_{c}=\frac{\left(n^{2}-1\right)}{12} \frac{E h^{3}}{r^{3}}=\frac{E}{4}\left(\frac{h}{r}\right)^{3},
$$

where $n$ indicates the buckling mode. Typically $n=2$ in real systems for the lowest critical buckling pressure. Here, $r$ is the tube radius, $h$ is the tube wall thickness, and $E$ is the Young's modulus of the material. Notice that only the ratio $h / r$ appears in the buckling condition, rather than either value individually. Details of the derivation of Eq. (5) can be found in Timoshenko and Gere ${ }^{28}$ or Brush and Almroth ${ }^{29}$ for the case of buckling of rings.

The ring buckling solution is used in this analysis because it can be applied to tubes with a free boundary condition at one end, which are hence unconstrained in the axial direction. For boundary conditions where the tube is constrained in the axial direction, a tube buckling solution should be used, which adds a factor of $1 /\left(1-v^{2}\right)$ in Eq. (5). Due to the relatively slow change in cross-sectional shape along the tube, it was approximated that the pressure at each plane perpendicular to the tube axis is directly related to the local tube deformation at that point.

For an elastocapillary interaction, a comparison of the critical buckling pressure given in Eq. (5), with the characteristic lateral pressure due to surface tension, $P_{\sigma}=2 \sigma / r$ for a cylindrical tube, gives a condition for elastocapillary buckling

$$
\left(\frac{\sigma r^{2}}{E h^{3}}\right) \geq \frac{1}{8} .
$$


TABLE I. Criteria for the transitions to buckling, opposite wall contact, and line contact in an elastocapillary interaction assuming a characteristic lateral pressure due to surface tension, $P_{\sigma}$. A Poisson's ratio of $v=0.5$ was used. Numerical values were converted from simulations by Flaherty et al. ${ }^{26}$

\begin{tabular}{lc}
\hline \hline Regime & Criteria \\
\hline Buckling to ellipse shape & $r / L_{e c} \geq 1.061$ \\
Opposite wall contact & $r / L_{e c} \geq 1.620$ \\
Line contact & $r / L_{e c} \geq 2.274$ \\
\hline \hline
\end{tabular}

Once a tube has buckled, it can be modeled using predictions from the literature for post-buckling behavior. Flaherty et al. ${ }^{26}$ give numerical results for the threshold pressure values for opposite wall contact, and for zero curvature contact, or the initial onset of line contact, for a ring or tube. Comparing these numerical predictions with the expected pressure due to surface tension gives the same scaling as Eq. (6), but with different numerical threshold values for each case, leading to dimensionless criteria of the same form as Eq. (6).

These shell theory predictions relate closely to the elastocapillary length used in the related literature. If one uses the tube radius as the relevant length scale, the ratio $r / L_{e c}$ can be squared to result in the same scaling as Eq. (6), and the numerical constants can be adjusted accordingly to give criteria in terms of the ratio of radius to elastocapillary length (see Eq. (1)). The resulting dimensionless criteria for buckling, opposite wall contact, and zero curvature contact or line contact, in terms of the elastocapillary length are given in Table I for the case of a Poisson's ratio of $v=0.5$ (incompressible). The tube radius is approximately equal to its elastocapillary length at the onset of buckling (Table I). Since the results in Table I are derived for a characteristic pressure based on a circular tube shape, they are expected to be accurate for the onset of buckling but only approximate for opposite wall contact and line contact due to the non-circular shape of the tube and meniscus at the onset of those states. This is discussed further in Sec. IV.

\section{EXPERIMENTS}

\section{A. Capillary flow in flexible tubes}

Capillary rise and evaporation experiments were performed using flexible tubes. Flexible tubes were manufactured using Elite Double 8 (Zhermack), a silicone-based material with an elastic modulus of approximately 0.2 MPa. This material was chosen for its low elastic modulus as well as its low viscosity before curing, which allowed for manufacturing of very thin-walled tubes to meet the criteria for deformation in the different regimes. Wires with a range of diameters $D=2 r$ were dip coated with this material, then held in a precisely vertical position during curing in order to manufacture very thin-walled tubes of a uniform wall thickness $h$ around the perimeter. Gravity-driven flow of the material during curing leads to some axial variation in wall thickness along the tube length; however, this was less important than the variation around the perimeter for these experiments. Due to variability in the manufacturing process, wall thickness was measured for each tube, and the flexible tubes with the most uniform wall thickness were chosen for the experiments.

The thicknesses of the tube walls were measured using a reflected light microscope to image either thin cross-sectional slices from each end of a tube or the ends of the tubes themselves. Examples of typical images of cross sections are shown in Figure 3. For the experiments, the tubes generally had less than $10 \%$ variation in wall thickness around the perimeter, while a variation of up to $25 \%$ was accepted for a few cases which tended to have more variability in manufacturing. When making the predictions of deformation, the smallest value of wall thickness around the perimeter was used.

Capillary-driven rise of liquid into these flexible tubes was first examined. The tubes were suspended from rigid metal tubes and held vertically, using rigid tubes whose outer diameter was equal to the inner diameter of the flexible tube. A liquid bath of isopropanol was carefully brought into contact with the end of the tube, and capillary rise into the tube was recorded from the side 


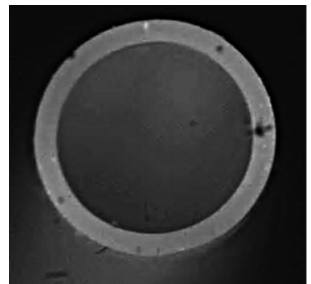

(a)

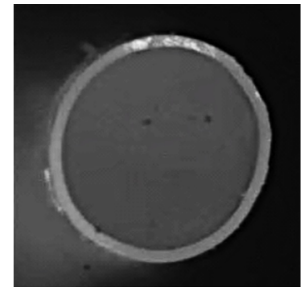

(b)

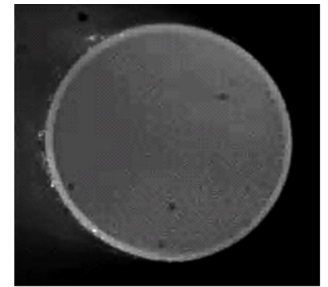

(c)

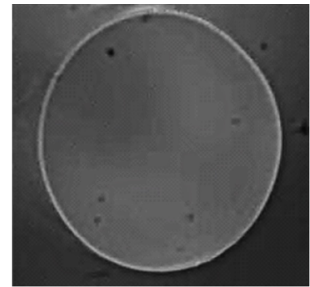

(d)

FIG. 3. Typical images of tube cross sections taken with a reflected light microscope, in order of decreasing ratio of wall thickness to radius. (a) $D=2 r=1.65 \mathrm{~mm}, h=40 \mu \mathrm{m}$, (b) $D=1.05 \mathrm{~mm}, h=30 \mu \mathrm{m}$, (c) $D=0.91 \mathrm{~mm}, h=26 \mu \mathrm{m}$, and (d) $D=1.65 \mathrm{~mm}, h=20 \mu \mathrm{m}$.

using a video camera (Thorlabs DCC1645C). Isopropanol was chosen as the liquid, because it is perfectly wetting on the flexible material, with a contact angle $\theta=0^{\circ}$.

A schematic of this experiment, including the expected final pressure distribution within the tube, is shown in Figure 4. The magnitude of the capillary pressure is the highest just below the liquid meniscus, acting inward on the tube. Due to hydrostatic variation, the pressure at the base of the tube is atmospheric. An example of images from a video of capillary rise is shown in Figure 5. As expected, the liquid velocity is initially higher, then decreases before the liquid reaches a final equilibrium height.

Some deformation was visible in these capillary rise experiments; however, the boundary conditions of the liquid meniscus at the base of the tube, on the outside of the tube, tended to prevent significant deformation. Also, the proximity of the top meniscus to the rigid tube would tend to prevent significant deformation. The persistence length ${ }^{30}$ for a pinch is on the order of $1 \mathrm{~cm}$ for the tubes used in these experiments, so the impact of the rigid tube may be significant. Additionally, axial variation in wall thickness inhibited deformation at the greater rise heights for some tubes and made the capillary rise experiments less useful for testing criteria of collapse and coalescence.

\section{B. Evaporation experiments}

Evaporation experiments were then performed with flexible tubes. In the evaporation experiments, a flexible tube was again suspended from a rigid metal tube and held vertically, where the outer diameter of the rigid tube was equal to the inner diameter of the flexible tube. A liquid bath was supplied at the base of the tube and liquid was allowed to rise into the tube through capillary

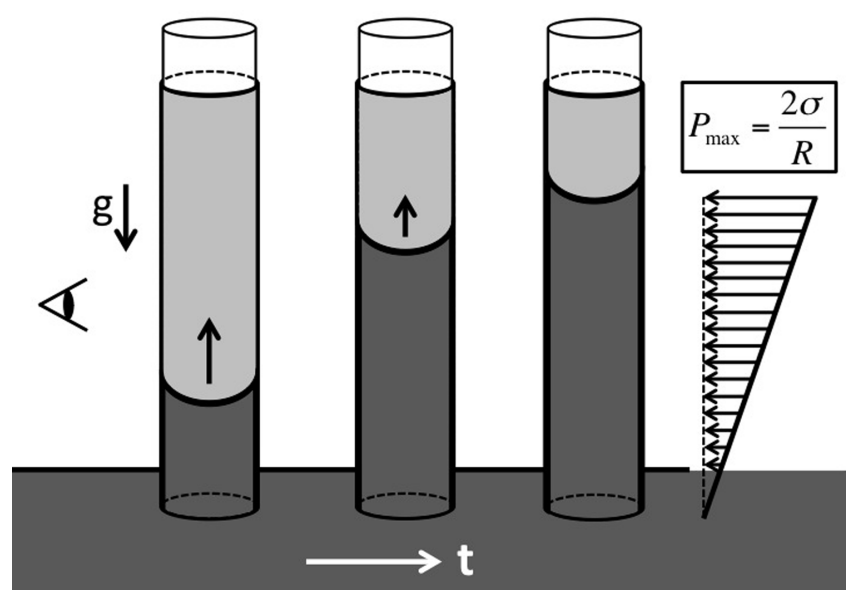

FIG. 4. Schematic of the capillary rise experiment. A flexible tube (light gray) is suspended vertically from a rigid metal tube (white) of the same diameter, which is open at the top. Liquid (dark gray) rises into the flexible tube through capillary action, first at higher velocities, then more slowly as the liquid column height increases, due to increasing viscous and gravitational resistance. The final equilibrium pressure distribution is shown on the right and varies hydrostatically. 


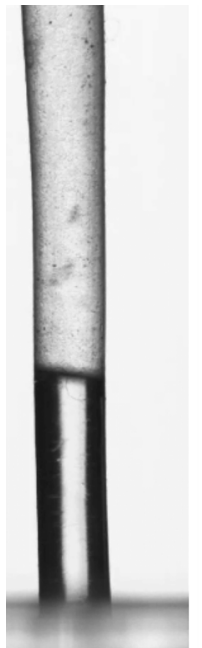

(a) $t<0.02 \mathrm{~s}$

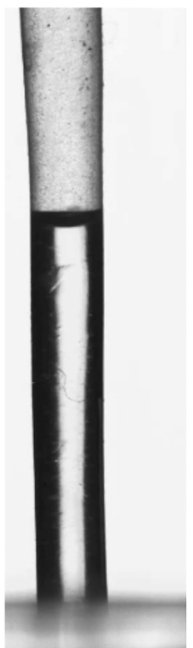

(b) $t=0.2 \mathrm{~s}$

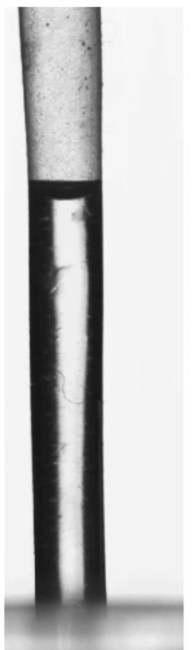

(c) $t=0.4 \mathrm{~s}$

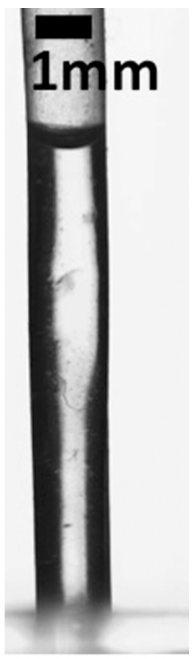

(d) $t=10 \mathrm{~s}$

FIG. 5. Images from a capillary rise experiment with a flexible tube. (a) $t<0.02 \mathrm{~s}$ after the liquid comes into contact with the flexible tube, (b) $t=0.2 \mathrm{~s}$, (c) $t=0.4 \mathrm{~s}$, and (d) $t=10 \mathrm{~s}$ shows the final equilibrium state. While there is some deformation during capillary rise, the boundary condition at the base of the tube prevented it from deforming further.

action to its equilibrium rise height, then the liquid bath was removed. Isopropanol was again used as the liquid because it evaporates relatively quickly, and is completely wetting on the flexible material. Tubes were soaked in isopropanol prior to each experiment so that the entire tube had been exposed to the liquid. This avoided localized swelling that would affect the deformation, without significantly impacting tube dimensions or steady-state properties.

The tube was then observed simultaneously from the side and from below during evaporation of the liquid, to measure the deformation of the tube due to capillary pressure (Figure 6). Side views allowed for measurement of the height of the liquid column, taken at the bottom of the liquid meniscus. The bottom views were used to assess the deformation regime, namely, circular, buckled, opposite wall contact or line contact, and to measure the final cross-sectional area $A$ and undeformed initial area $A_{0}$ of the tube at the bottom end using a Matlab routine.

For tubes with a final state in the line contact regime, the cross sectional area $A$ was also measured for the transition points to opposite wall contact and line contact. The height of the liquid column at these transition points $Z_{T}$ was determined from the side view video of the tube at the same time. The height was then used to find the pressure at the base of the tube at the transition points.

The metal tube holding the flexible tube is open, such that evaporation of the liquid occurs from both sides. The liquid remains at the lower end of the tube throughout the experiment due to gravity.

This experimental setup allows for a free boundary condition at the bottom of the tube, leading to more significant deformation that can be measured at the base of the tube. The vertical configuration avoids gravity effects across the tube that might impact its buckling and deformation by symmetry breaking. The liquid pressure varies hydrostatically within the tube. However, as the liquid evaporates, this variation becomes smaller and the bottom of the tube experiences a more uniform pressure very close to the maximum capillary pressure (see Figure 6(b)). Also, as the axial distance covered by the liquid becomes smaller, the axial variation in tube wall thickness becomes less significant and the tube can be modeled as having a single wall thickness equal to its wall thickness at its end.

Images from an initial vertical evaporation experiment in which a tube containing liquid deforms under capillary pressure are shown in Figure 7. This tube has non-uniform wall thickness, leading to interesting twisting deformations and meniscus shapes. Although this flexible tube is the same tube shown in Figure 5, deformation under capillary pressures was much more significant in the case of vertical evaporation, due to the free boundary condition at the end of the tube. There 

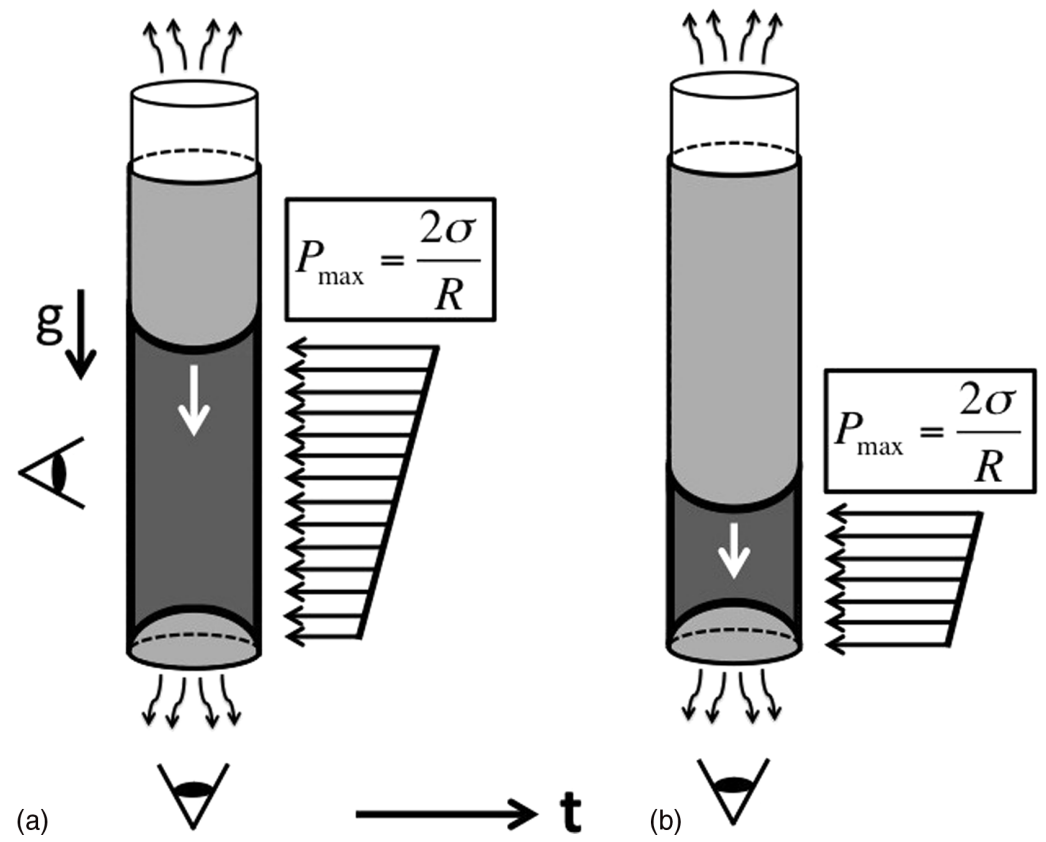

(b)

FIG. 6. Evaporation experiment. A flexible tube (light gray) is suspended vertically from a rigid metal tube (white) of the same diameter, which is open at the top. (a) Liquid (dark gray) is allowed to rise into the tube initially, then evaporates. The flexible tube is imaged from the side and from below during evaporation to measure deformation. Note the free boundary condition at the base of the tube, and the vertical configuration which prevents gravitationally driven deformation across the tube. (b) As the liquid evaporates, hydrostatic variations in pressure become less significant, leading to a more uniform pressure approximately equal to the maximum capillary pressure.

is also a stronger capillary pressure near the end of the tube due to the shorter liquid plug, which minimizes hydrostatic variation, and the fact that there is a meniscus on each side of the liquid rather than a connection to a liquid bath (see Figure 6).

For these reasons, evaporation allows for more significant deformation, and was studied in more detail than capillary rise.

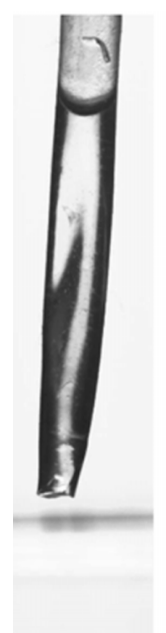

(a) $t=0 \mathrm{~s}$

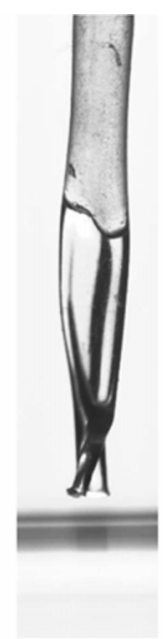

(b) $t=14 \mathrm{~min}$

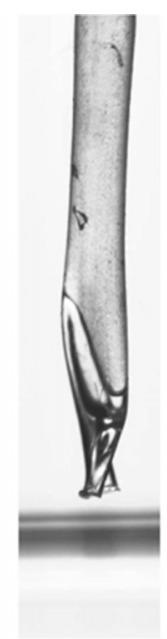

(c) $t=19 \mathrm{~min}$

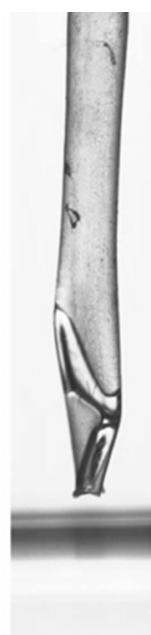

(d) $t=22 \mathrm{~min}$

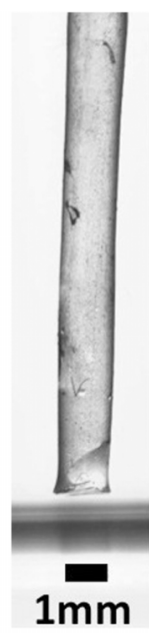

(e) $t=24 \mathrm{~min}$

FIG. 7. Example of a vertical evaporation experiment in a flexible tube suspended from a rigid metal tube. In (a) through (d), the liquid evaporates, deforming the tube; in (e), the tube reverts to its initial cylindrical shape. This tube does not have uniform wall thickness, leading to unusual twisting deformations. This is the same flexible tube used in Figure 5; however, deformation is much more significant due to the free boundary condition at the base. 
TABLE II. Experimental parameters for evaporation experiments in flexible tubes.

\begin{tabular}{lc}
\hline \hline Experimental parameter & Range of values \\
\hline Tube inner diameter $(I D=2 r)$ & $I D=0.65-1.65 \mathrm{~mm}$ \\
Tube wall thickness $(h)$ & $h=16-76 \mu \mathrm{m}$ \\
Tube Young's modulus $(E)$ & $E=0.2 \mathrm{MPa}$ \\
Liquid surface tension $(\sigma)$ & $\sigma=21 \mathrm{mN} / \mathrm{m}$ \\
\hline \hline
\end{tabular}

\section{Experimental parameters}

Table II lists the range of experimental parameters used for the evaporation experiments. A range of tube diameters and wall thicknesses was tested to meet the expected criteria for $r / L_{e c}$ for the four different regimes (Table I).

In order for capillary effects to dominate over gravity, allowing for spontaneous capillary deformation of the tube and negligible gravity effects, the characteristic length scale or the tube radius, must be less than the capillary length of the liquid (Eq. (2)). Thus, both the tube radius and the wall thickness to radius ratio must be relatively small, leading to a fairly restrictive parameter range for spontaneous capillary deformation.

The threshold $r=L_{\text {cap }}$ is shown graphically in Figure 8. For isopropanol, the liquid used in the experiments, the capillary length is approximately $L_{c a p}=1.5 \mathrm{~mm}$.

\section{RESULTS AND DISCUSSION}

The observed deformations of the evaporation experiment were compared with theoretical predictions. The view from below the tube was used to determine which regime was observed, namely, whether the tube had remained circular, buckled, contacted at a point, or collapsed. The image used to determine the result was the final frame in which the tube cross section contained liquid, since the capillary pressure becomes more uniform and gravity effects and variations in wall thickness become less important as the height of the liquid column decreases. The final area of the collapsed tube was also measured for several data points.

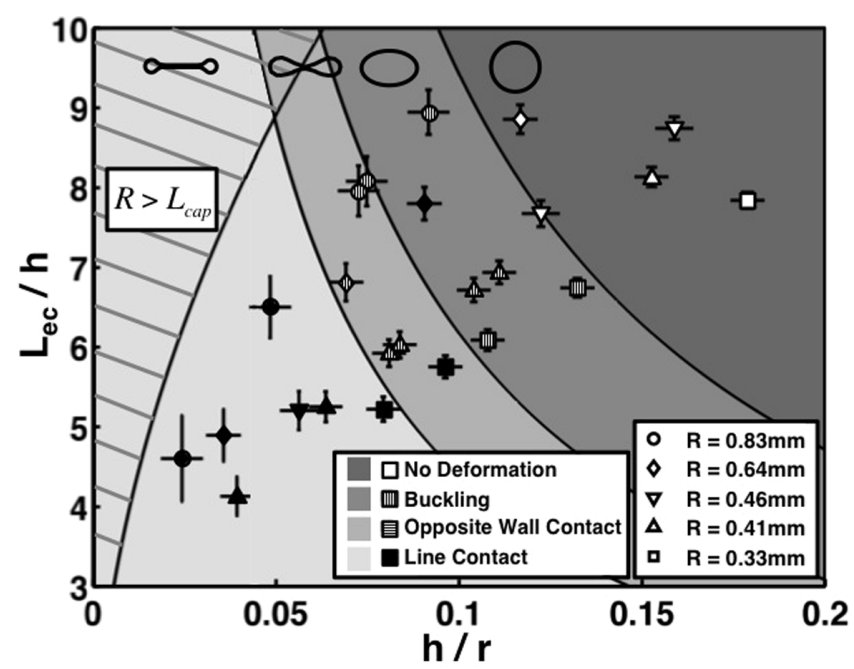

FIG. 8. Regime map of tube deformation showing predicted and experimental results. The location of data points on the plot is set by the tube material, geometry, and liquid surface tension. Shape indicates the radius of the tube. The background color indicates the predicted behavior, and the shading of each point gives the observed behavior. Note that the case of opposite wall contact was not observed in these experiments. The area to the left of the line with positive slope shows where the radius is greater than the capillary length of the liquid and gravity effects begin to dominate. 
As discussed above, the theory predicts four regimes: a state where the tube remains circular, a buckled state, opposite wall contact, and line contact. This theoretical regime map as well as the experimental results are shown in Figure 8, which shows normalized elastocapillary length, $L_{e c} / h$, versus the tube wall thickness to radius ratio, $h / r$. The tube material, geometry, and liquid surface tension fix the location of each data point on the plot, and the symbol shape corresponds to the tube radius. The background color indicates the predicted behavior, and the shading or patterning of each point gives the observed behavior. Error bars indicate the estimated uncertainty. Since uncertainty primarily arises from uncertainty in the measurement of wall thickness, error bars are based solely on uncertainty in $h$, approximated as two pixel widths in the images used for wall thickness measurements. Other factors including uncertainty in the radius and elastic modulus also contribute to uncertainty, but proved to be less significant and are not included in Figure 8.

The line with positive slope indicates the threshold, where the radius is equal to the capillary length of the liquid (Eq. (2)). The gravity-dominated regime to the left of this line was avoided. On the lower right hand side of the plot, experiments were limited by manufacturing and measurement considerations for the very small tube diameters.

The results shown in Figure 8 demonstrate that, overall, the thresholds between different regimes are well predicted by the theory, for the initial onset of buckling and the case of line contact or coalescence.

However, more scatter was observed in the middle transition regimes, and experimentally, only three of the regimes were observed for the end state of the vertical evaporation experiment. The case of opposite wall contact, without coalescence, was not observed as a final equilibrium state; however, it was observed during the transition to line contact.

Some deviation from the simple model is to be expected in the opposite wall contact regime since the meniscus shape changes significantly as the tube walls approach one another. The driving pressure is dominated by the smaller length scale between the two sides of the tube that are almost in contact, so this concave shape might be expected to become unstable and transition directly to line contact. Additionally, adhesion forces between the sides of the flexible tube could play a role at close range. However, in the experiments, tubes in those regimes were found to buckle, but stay open, so there was no contact between opposite sides.

The data points in question were repeated with tubes in a horizontal configuration, again with one free end, to investigate whether gravitational effects may play a role. However, there was no significant difference in the final state of the tubes between the horizontal and vertical experiments.

A possible explanation for this deviation from the theory arises from the fact that the wall thickness to radius ratios of the tubes in the buckling and opposite wall contact regimes are relatively large, due to the gravitational size constraints, making the shell theory approximation less accurate. When $h / r$ is larger, stress is expected to vary with radius within the tube walls, and
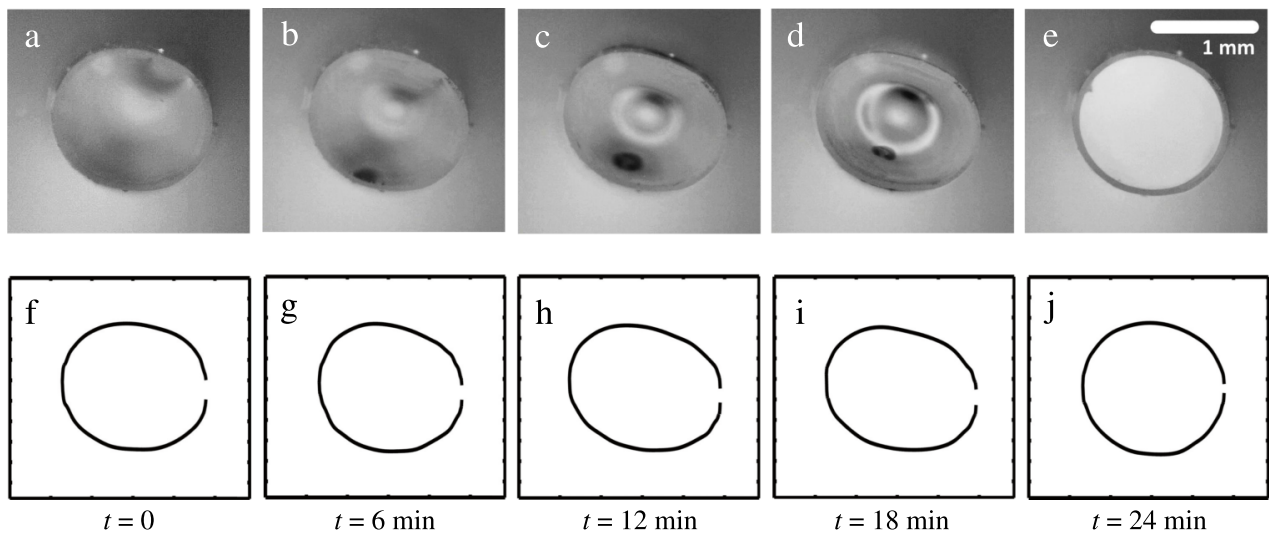

FIG. 9. (a)-(e) Series of bottom views in the buckling regime ( $r=0.64 \mathrm{~mm}, h / r=0.069)$, inverted gray scale, and extracted contours (f)-(j) at given times during an evaporation experiment. These contours were used to calculate the areas of the deformed tube, $A$, and the reference area of the undeformed tube, $A_{0}$. 
thick-walled tubes are not expected to buckle or have the same post-buckling behavior described by shell theory.

However, achieving tubes with low $h / r$ values requires moving to the left on the regime map (Figure 8), and to remain in the buckling and opposite wall contact regimes would require tubes with fairly large radii, approaching or exceeding the capillary length.

Fortunately, an alternative method of viewing these regimes was found. Many of the flexible tubes in the line contact regime had thin enough walls that $h / r$ was quite small $(<8 \%)$, and therefore, they did follow the expected pattern of deformation and pass through each of the stages during the course of the evaporation experiment.

Three test cases in the line contact regime were chosen and studied in more detail. During the course of the evaporation experiments, these were found to pass through all three of the deformed regimes. Buckling typically happened immediately, but opposite wall contact and line contact occurred at discrete measurable times during evaporation. Thus, two transition points could be observed using the video from below the tube: transition to opposite wall point contact and transition to zero curvature contact (initial line contact). Typical images of the tube deformation over time during evaporation are shown in Figures 9 and 10 for the cases of buckling and line contact, respectively.

As shown in these figures, the deformation during evaporation takes place slowly, with time scales on the order of minutes. For the case of line contact (Figure 10), the opposite walls of the tube initially contact at a point, then this point of contact reaches zero curvature and spreads into a line. The final shape has contact between the two sides over a line in the center with two small open areas at the edges.

As discussed in Sec. III, the liquid heights and deformed areas were measured at the time of each transition point in order to find the capillary pressure difference at the bottom of the tube. It was assumed that at the top liquid meniscus, the tube remained circular, which was a good approximation for these cases. In addition to experimental observation, this approximation can be justified with the concept of a persistence length, as described by Mahadevan et al..$^{30}$ The persistence length for the tubes used in these experiments is on the order of $1 \mathrm{~cm}$, which is comparable to the liquid height during most of the experiment. The distance at which a cross section would be different enough from a circle to substantially affect the meniscus curvature would be even shorter.

Using this assumption, the capillary pressure at the base of the tube can be calculated from the hydrostatic pressure variation

$$
P^{\star}=\frac{P-P_{a}}{K_{p}}=-\frac{\left(\frac{2 \sigma}{r}-\rho g Z_{T}\right)}{K_{p}} .
$$

Here, $Z_{T}$ is the height of the liquid column at the transition point to either opposite wall contact or line contact.
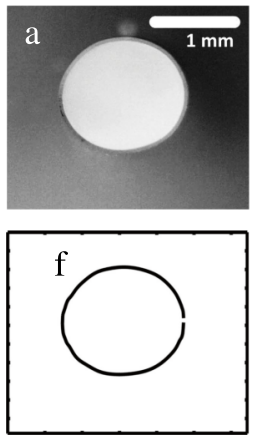

Initial
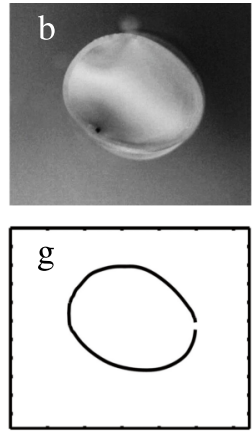

$t=0$
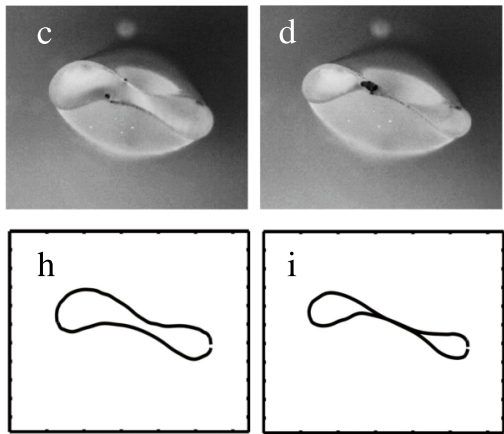

$t=150 \mathrm{~s}$

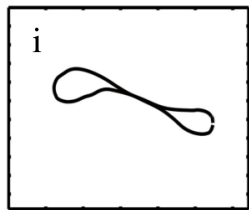

$t=300 \mathrm{~s}$
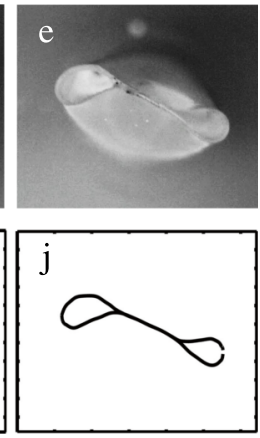

$t=600 \mathrm{~s}$

FIG. 10. (a)-(e) Series of bottom views in the line contact regime ( $r=0.64 \mathrm{~mm}, h / r=0.036)$, inverted gray scale, and extracted contours (f)-(j) at given times during an evaporation experiment. These contours were used to calculate the areas of the deformed tube, $A$, and the reference area of the undeformed tube, $A_{0}$. In this regime, opposite walls of the tube initially touch at a point, which then grow to a line of contact. 
These results for normalized pressure $P^{*}$ and area $A / A_{0}$ at the contact and line contact transitions are shown in Figure 11 in the darker gray (blue) and lighter gray (red) symbols, respectively. The results for the final deformed states of tubes are also included in this figure. For these cases, the capillary pressure difference was simply modeled as

$$
P^{\star}=\frac{P-P_{a}}{K_{p}}=-\frac{2 \sigma / r}{K_{p}}=-\frac{2 r^{2}}{L_{e c}^{2}} .
$$

The shell theory predictions for normalized pressure and area at the transition points are indicated for comparison. The similarity solution for the line contact regime is shown in the solid black line, and the predicted transition pressures for buckling, opposite wall contact, and line contact are shown with dashed lines.

As seen in this graph, for the results from the line contact videos, the observed transition pressures are well predicted by the theoretical values for all but one of the cases. The area ratios, however, differed significantly from the expected values represented by the line in Figure 11, and in some cases could not be measured precisely. Tubes with final states in the buckling regime did not show the expected behavior, likely due to the larger wall thickness to radius ratios.

Uncertainty in normalized pressure was again calculated from the uncertainty in the wall thickness value, which is the primary source of error. Uncertainty in the normalized area was based on the percentage error in normalized area for the cases which did not buckle, and is expected to have a value of $A / A_{0}=1$. This was assumed to give an indication of uncertainty in the area measurements.

Figures 12 and 13 show the shapes of the tubes that reached line contact, at the transition points to opposite wall contact (Figure 12) and line contact (Figure 13). These are shown in comparison with a schematic of the numerically simulated shape found by Flaherty et al. ${ }^{26}$ The white lines on the images indicate the shape that was extracted from the image and used to calculate the area. The tube that differs the most from the theoretically predicted shape, Figures 12(d) and 13(d), also has the largest wall thickness to radius ratio $h / r$.

As mentioned in Sec. II, predictions of the simple model are expected to be less accurate as the tube deforms because of the variation in capillary pressure within the tube, caused by its deformation. A complete description of this nonlinear problem is beyond the scope of the current work; however, to gain further insight into experimental results and to further explore the characteristics of

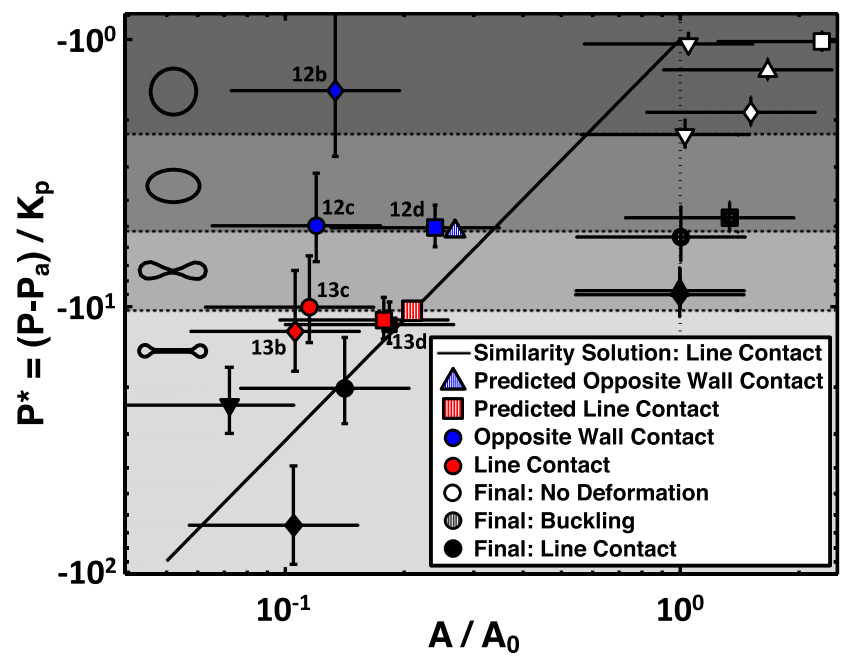

FIG. 11. Normalized capillary pressure versus normalized area: comparison of theory and experiments. The solid black line represents the similarity solution given by Flaherty et al. ${ }^{26}$ for the line contact regime. The darker gray (blue) and lighter gray (red) data points correspond to transitions within the line contact videos for three test cases as shown in Figures 12 and 13 , while the other data points are final states. Color indicates the observed regime, while the shape indicates the tube size in the same manner as Figure 8. The characteristic applied capillary pressure is normalized by $K_{p}$ as shown in Eqs. (7) and (8). Note that the tubes in the buckling regime did not show the expected behavior, likely due to the larger ratio of wall thickness to radius. 


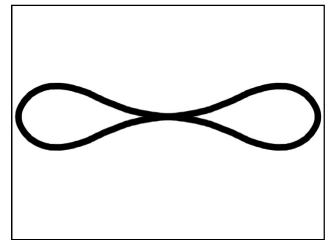

(a)

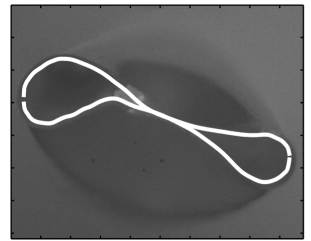

(b)

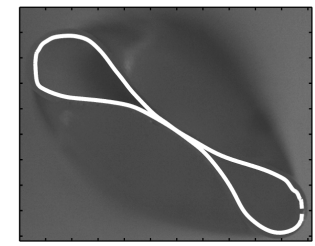

(c)

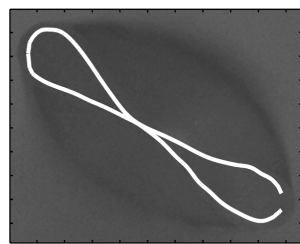

(d)

FIG. 12. Opposite wall contact. (a) Numerically predicted shape for opposite wall contact. $^{26}$ (b) and (c): Experimental images of the transition to opposite wall contact for three test cases which then continued into line contact. The white line indicates the shape that was used to calculate the area. (b) $r=0.64 \mathrm{~mm}, h / r=0.036$. (c) $r=0.83 \mathrm{~mm}, h / r=0.048$. (d) $r=0.33 \mathrm{~mm}, h / r=0.079$.

the system, this variation in capillary pressure was examined for idealized deformed shapes. Figure 14 shows capillary pressure versus area for simple models of the deformed tube shapes. Values are normalized by the capillary pressure and area in the circular tube, $P_{0}$ and $A_{0}$, respectively, and it was assumed that the total length of the perimeter of the cross section remains constant, which is a good approximation for this situation. ${ }^{26}$

In the initial circular shape, the magnitude of the capillary pressure is

$$
P_{0}=\frac{2 \sigma}{r} \text {. }
$$

This is shown on the far right of Figure 14. As the tube deforms to an ellipse, the total capillary pressure changes and it can be approximated as

$$
P_{\text {ellipse }}=\sigma\left(\frac{1}{a}+\frac{1}{b}\right),
$$

where $a$ and $b$ are half the major and minor axes of the ellipse. For the assumption of constant perimeter, the capillary pressure of an ellipse increases monotonically during deformation to a smaller area. This is shown in the dashed-dotted line of Figure 14, and an experiment would follow this line to the left.

After the ellipse shape, there is an intermediate regime shown in the solid black line in Figure 14. This configuration is represented by two semicircles of constant radius connected by diagonal lines which approach a point of contact. In this intermediate regime, once the shape becomes concave and opposite walls begin to approach each other at a point, the pressure across the gap increases continuously with the inverse of the gap distance

$$
P_{\text {concave }}=\frac{1}{\delta},
$$

where $\delta$ is the distance between the two corner points.

However, the pressure in the outer lobes of the shape remains similar to the ellipse pressure. Because of this difference in pressure, flow is driven within the tube cross section until the pressure stabilizes. This unstable arrangement may help explain why the intermediate stage of opposite wall contact is not seen in the experiments as a final equilibrium state.

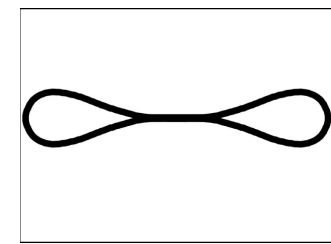

(a)

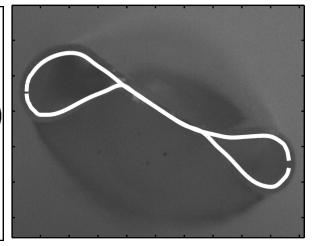

(b)

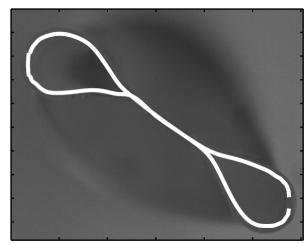

(c)

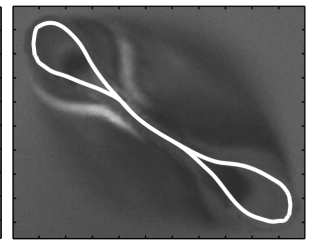

(d)

FIG. 13. Line contact. (a) Numerically predicted shape for initial line contact. ${ }^{26}$ (b) and (c): Experimental images of the transition to line contact for three test cases which continued into line contact. The white line indicates the shape that was used to calculate the area. (b) $r=0.64 \mathrm{~mm}, h / r=0.036$. (c) $r=0.83 \mathrm{~mm}, h / r=0.048$. (d) $r=0.33 \mathrm{~mm}, h / r=0.079$. 


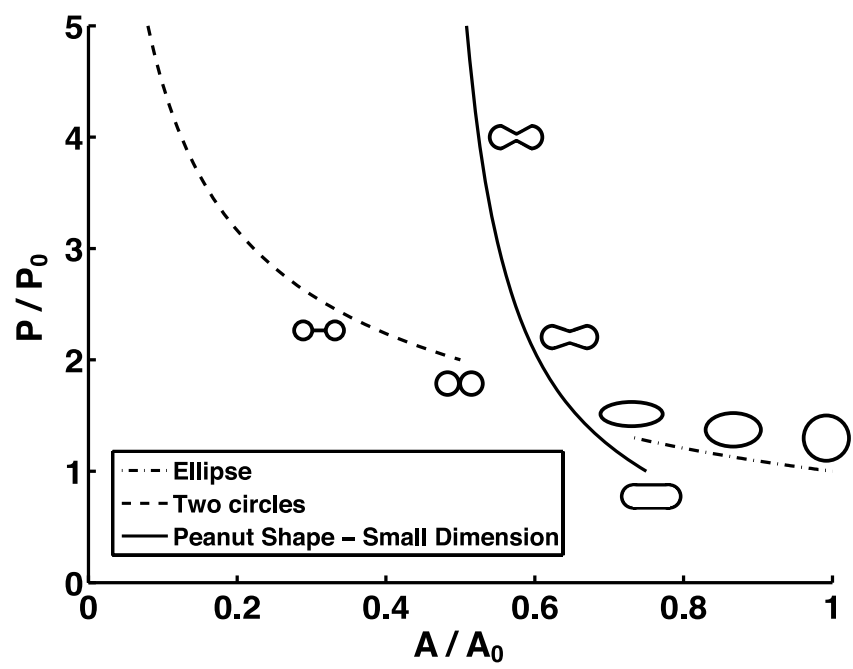

FIG. 14. Capillary pressure versus cross sectional area for a range of idealized shapes for deformed tubes. Note that in the intermediate regime, the capillary pressure drop in the small gap is expected to be high due to the small length scale and high curvature, while the pressure drop in the outer lobes will remain similar to the ellipse pressure. Thus, there is a capillary force pulling liquid into the gap from the outer circles, and this may make the opposite wall contact case unstable and more difficult to see experimentally. However, since the contact regime was seen during transition and appears similar to the shapes for uniform lateral pressure, experimental results suggest that the meniscus adjusts so that the pressure actually remains more uniform over the cross section.

Opposite wall contact is, however, seen as a temporary stage during the experiments in the line contact regime, and the shape formed by the tube is quite similar to the theoretical shape for a uniform lateral pressure, as shown in Figures 12 and 13. This indicates that the meniscus curvature adjusts to maintain a constant pressure at the bottom plane during these experiments, rather than varying with horizontal location in the cross-section. Thus, the regime indicated by the solid line may not actually be seen experimentally. The fact that opposite wall contact was not observed as a final state is likely due to other factors, such as the length and boundary conditions of the tubes, imperfections, or circumstantial effects due to the size constraints and the larger wall thickness to radius ratio in those cases, as described above.

Once opposite wall contact and line contact have occurred, the capillary pressure again increases continuously for the line contact regime, and this is shown for the simple model of two circles connected by a line, with the length of the line increasing and the radius of the two circles decreasing correspondingly to maintain a constant perimeter.

$$
P_{2 \text { circles }}=\frac{2 \sigma}{r_{\text {small }}} .
$$

In an experiment starting from a circular tube, the capillary pressure would be expected to follow the dashed-dotted line for deformation to an ellipse, then there would be a complex interaction in the intermediate regime that maintains the pressure close to the final ellipse pressure, and finally the pressure would increase again for the line contact configuration moving to the left along the dashed line. With these idealized shapes, the capillary pressure is predicted to be within a reasonable factor of the characteristic pressure $P_{0}$ up until the point of line contact.

In summary, this study has demonstrated that capillary forces can cause sufficiently flexible tubes to deform or coalesce. A regime map was developed for the different stages of tube deformation, and it was shown that the extreme cases, of complete coalescence and initial onset of deformation, are well predicted by classical shell theory using a characteristic Laplace pressure. The intermediate stages, however, were more complex.

For coalescence of flexible tubes to occur spontaneously due to surface tension, a fairly restrictive set of conditions must be met, since the tube radius must be small enough for capillary forces to dominate, while the wall thickness to radius ratio $h / r$ must also be low, thus requiring very small, thin-walled tubes. 
It was found that despite the three-dimensional effects and non-axisymmetric buckling involved in deformation of flexible tubes, the elastocapillary length is still useful for modeling this problem, if an appropriate length scale-in this case the tube radius-is chosen. These results are encouraging for the use of these concepts in detailed modeling of capillary flows in deformable porous media and other complex structures.

\section{ACKNOWLEDGMENTS}

T. Hoberg gratefully acknowledges support from a National Defense Science and Engineering Graduate (NDSEG) Fellowship.

\section{APPENDIX: REVIEW OF CAPILLARY RISE IN RIGID TUBES}

The pressure difference across a liquid meniscus, due to surface tension, is given by Eq. (A1), with $R_{1}$ and $R_{2}$ the two radii of curvature of the liquid meniscus, and $\sigma$ the surface tension of the liquid

$$
\Delta P=\sigma\left(\frac{1}{R_{1}}+\frac{1}{R_{2}}\right)
$$

For rise of a liquid into a small tube driven by surface tension, or capillary rise, balancing hydrostatic and capillary pressure predicts that the liquid will reach a final height that is inversely proportional to the tube dimensions. Considering two cases, a circular tube with radius $r$, and a gap between two sheets with gap width $b$, the driving capillary pressure and equilibrium rise height are given by

$$
\Delta P=\frac{2 \sigma}{L} \quad Z_{\text {final }}=\frac{2 \sigma}{\rho g L} .
$$

Here, $L=b$ for a gap between two sheets, $L=r$ for a tube, and $Z_{\text {final }}$ is the final equilibrium height of the meniscus.

During capillary rise, balancing the driving pressure of surface tension with viscous and gravitational forces gives the evolution equation for the dynamics of the meniscus height as a function of time. ${ }^{25}$ Neglecting inertial effects yields

$$
\frac{2 \sigma \cos (\theta)}{L}=\frac{c_{1} \mu z \dot{z}}{L^{2}}+\rho g z .
$$

For two sheets, $c_{1}=12$, and for a tube, $c_{1}=8$. Here, $\mu$ is the dynamic viscosity of the liquid, $z$ is the liquid height, and $\theta$ is the contact angle of the liquid on the surface.

In the early time limit where gravity can be neglected, we find the classical height dependence on the square root of time, $h \sim \sqrt{t}$, and the velocity of the fluid is inversely proportional to the square root of time. At later times, an exponential decay to the final height is predicted. An initial capillary rise dominated by inertia can also be included, ${ }^{31}$ which gives a linear relationship between height and time; however, this was not significant in the current experiments and thus inertia has been neglected. Specifically

$$
\operatorname{Re}=\frac{\rho \dot{z} L}{\mu} \ll 1 \text { for } t>t_{i v} \sim \rho L^{2} / \mu,
$$

where $R e$ is the Reynolds number and $t_{i v}$ is the time of transition between inertial and viscous regimes, ${ }^{25}$ which is on the order of milliseconds or less for these experiments, compared to the total time of several minutes.

\section{Preliminary experiments: Rigid tubes}

Preliminary capillary rise experiments were performed using rigid rectangular glass tubes, to test our experimental method and verify agreement between experiments and theory in this simple case. Glass tubes were carefully brought into contact with a liquid bath, and capillary rise was 


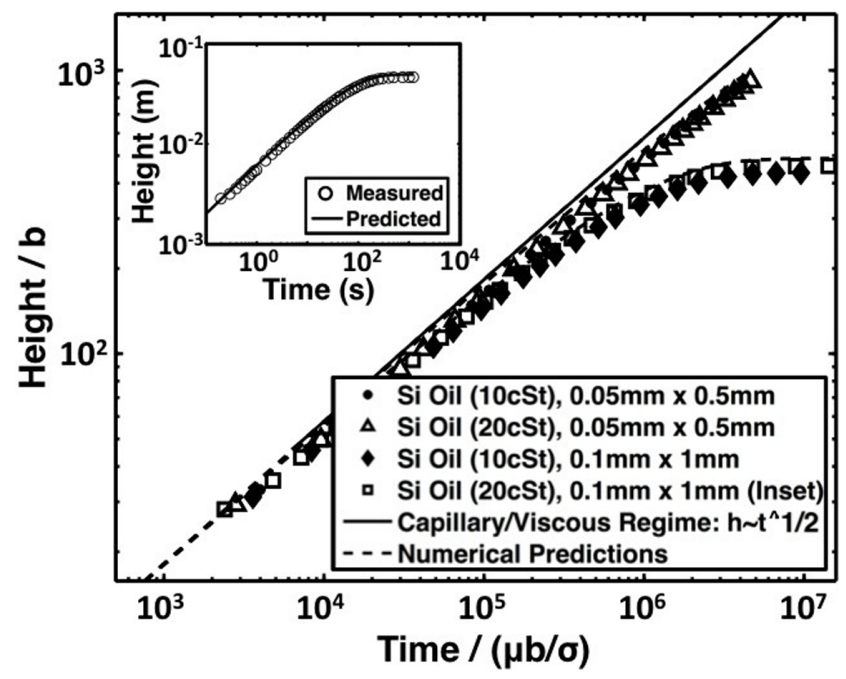

FIG. 15. Normalized rise height versus time for capillary rise in rigid tubes, varying the viscosity of the liquid and the tube dimensions. Height and time are normalized for the viscous regime. Inset: Example of dimensional rise height versus time for capillary rise in a rigid tube. The liquid is silicone oil $(v=20 \mathrm{cSt}, \sigma=0.021 \mathrm{~N} / \mathrm{m})$, and the tube dimensions are $0.1 \mathrm{~mm}$ $\mathrm{x} 1 \mathrm{~mm}$.

observed and recorded using a high definition video camera (Casio EX-F1). Results of rise height versus time were extracted from the videos and compared with the theoretically predicted values, calculated numerically from the evolution equation, Eq. (A3).

Data were taken for two viscosities of silicone oil $(v=10 c S t, 20 c S t)$ and for rectangular glass tubes of two different sizes $(0.1 \mathrm{~mm} \times 1.0 \mathrm{~mm}, 0.05 \mathrm{~mm} \times 0.5 \mathrm{~mm}$, VitroCom, Borosilicate). For these experimental parameters, viscous effects were dominant from early times and inertial effects were negligible. Because the tubes were rectangular, instead of the idealized case of a gap between two infinite sheets, there was a second radius of curvature in the plane of the plates, and a correction factor was used to account for this in the simulation. Thus, numerical predictions are based on Eq. (A3) with $c_{1}=12$ and with the surface tension term $2 \sigma / b$ replaced by $\sigma\left(\frac{2}{b}+\frac{1}{R_{2}}\right)$, where $R_{2}$ is half of the longer dimension of the rectangular cross section. A contact angle of $\theta=0^{\circ}$ was used in the predictions, consistent with the case of perfect wetting.

Typical results are shown in the inset of Figure 15, which shows experimental measurements and numerical predictions for silicone oil $(v=20 c S t)$ rising in a glass tube with a rectangular cross section of dimensions $0.1 \mathrm{~mm} \times 1 \mathrm{~mm}$. Normalized height over time is shown in Figure 15, with height scaled by the characteristic length, $b$, and time scaled by the characteristic capillary-viscous time, $t / t_{c}=\frac{\sigma \cos (\theta) t}{\mu b}$. As shown, data can be collapsed in the viscous regime using these scalings. Numerical simulations are shown as dashed lines. All physical parameters are known; hence, there are no fitting parameters in the simulation.

In the viscous regime, Eq. (A3) can be solved analytically yielding

$$
\frac{h}{b}=\left(\frac{t}{3 t_{c}}\right)^{1 / 2} \text {. }
$$

This curve is shown as the solid black line in Figure 15. Similarly, in the gravity-dominated exponential regime, data can be collapsed using different scaling parameters (not shown).

${ }^{1}$ T. Tanaka, M. Morigami, and N. Atoda, “Mechanism of resist pattern collapse during development process,” Jpn. J. Appl. Phys. Part 1 32, 6059-6064 (1993).

${ }^{2}$ C. Py, P. Reverdy, L. Doppler, J. Bico, B. Roman, and C. Baroud, "Capillary origami: Spontaneous wrapping of a droplet with an elastic sheet," Phys. Rev. Lett. 98, 156103 (2007).

${ }^{3}$ B. Pokroy, S. Kang, L. Mahadevan, and J. Aizenberg, "Self-organization of a mesoscale bristle into ordered, hierarchical helical assemblies," Science 323, 237-240 (2009).

${ }^{4}$ D. Halpern and J. B. Grotberg, "Fluid-elastic instabilities of liquid-lined flexible tubes," J. Fluid Mech. 244, 615-632 (1992). 
${ }^{5}$ A. L. Hazel and M. Heil, "Surface-tension-induced buckling of liquid-lined elastic tubes: A model for pulmonary airway closure," Proc. R. Soc. A 461, 1847-1868 (2005).

${ }^{6}$ A. L. Hazel and M. Heil, "Three-dimensional airway reopening: The steady propagation of a semi-infinite bubble into a buckled elastic tube," J. Fluid Mech. 478, 47-70 (2003).

${ }^{7}$ S. Kang, B. Pokroy, L. Mahadevan, and J. Aizenberg, "Control of shape and size of nanopillar assembly by adhesionmediated elastocapillary interaction," ACS Nano 4, 6323-6331 (2012).

${ }^{8}$ B. Roman and J. Bico, "Elasto-capillarity: Deforming an elastic structure with a liquid droplet," J. Phys.: Condens. Matter 22, 493101 (2010).

${ }^{9}$ H.-Y. Kim and L. Mahadevan, "Capillary rise between elastic sheets," J. Fluid Mech. 548, 141-150 (2006).

10 J. Bico, B. Roman, L. Moulin, and A. Boudaoud, “Adhesion: Elastocapillary coalescence in wet hair," Nature 432, 690 (1986).

11 J. van Honschoten, M. Escalante, N. Tas, H. Jansen, and M. Elwenspoek, "Elastocapillary filling of deformable nanochannels," J. Appl. Phys. 101, 094310 (2007).

${ }^{12}$ T. Cambau, J. Bico, and E. Reyssat, "Capillary rise between flexible walls," Europhys. Lett. 96, 24001 (2011).

13 J. M. Aristoff, C. Duprat, and H. A. Stone, "Elastocapillary imbibition,” Int. J. Non-Linear Mech. 46, 648-656 (2011).

${ }^{14}$ C. Duprat, J. Aristoff, and H. Stone, "Dynamics of elastocapillary rise," J. Fluid Mech. 679, 641-654 (2011).

${ }^{15}$ D. P. Gaver III, D. Halpern, O. Jensen, and J. Grotberg, "The steady motion of a semi-infinite bubble through a flexible-walled channel," J. Fluid Mech. 319, 25-65 (1996).

${ }^{16}$ J. Grotberg and O. Jensen, "Biofluid mechanics in flexible tubes," Annu. Rev. Fluid Mech. 36, 121-147 (2004).

17 A. Shapiro, "Steady flow in collapsible tubes," J. Biomech. Eng. 99, 126-147 (1977).

${ }^{18}$ M. Heil and A. L. Hazel, "Fluid-structure interaction in internal physiological flows," Annu. Rev. Fluid Mech. 43, 141-162 (2011).

19 A. Juel and A. Heap, “The reopening of a collapsed fluid-filled elastic tube," J. Fluid Mech. 572, 287-310 (2007).

${ }^{20}$ A. Heap and A. Juel, "Anomalous bubble propagation in elastic tubes," Phys. Fluids 20, 081702 (2008).

${ }^{21}$ Y. Yang, Y. Gao, D. Sun, M. Asta, and J. Hoyt, "Capillary force induced structural deformation in liquid infiltrated elastic circular tubes,” Phys. Rev. B 81, 241407 (2010).

${ }^{22}$ L. Preziosi, D. Joseph, and G. Beavers, "Infiltration of initially dry, deformable porous media,” Int. J. Multiphase Flow 22 , 1205-1222 (1996).

${ }^{23}$ J. Somner and A. Mortensen, "Forced unidirectional infiltration of deformable porous media," J. Fluid Mech. 311, 193-217 (1996).

${ }^{24}$ J. Siddique, D. Anderson, and A. Bondarev, "Capillary rise of a liquid into a deformable porous material," Phys. Fluids 21, 013106 (2009).

${ }^{25}$ N. Fries and M. Dreyer, "The transition from inertial to viscous flow in capillary rise,” J. Colloid Interface Sci. 327, 125-128 (2008).

${ }^{26}$ J. Flaherty, J. Keller, and S. Rubinow, "Post buckling behavior of elastic tubes and rings with opposite sides in contact," SIAM J. Appl. Math. 23, 446-455 (1972).

${ }^{27}$ D. Halpern, S. Naire, O. Jensen, and D. P. Gaver III, "Unsteady bubble propagation in a flexible channel: Predictions of a viscous stick-slip instability," J. Fluid Mech. 528, 53-86 (2005).

${ }^{28}$ S. Timoshenko and J. Gere, Theory of Elastic Stability (McGraw-Hill Book Company, Inc., 1961).

${ }^{29}$ D. Brush and B. Almroth, Buckling of Bars, Plates, and Shells (McGraw-Hill Book Company, 1975).

${ }^{30}$ L. Mahadevan, A. Vaziri, and M. Das, "Persistence of a pinch in a pipe," Europhys. Lett. 77, 40003 (2007)

${ }^{31}$ D. Quere, "Inertial capillarity,” Europhys. Lett. 39, 533-538 (1997). 